\title{
Diplejía facial aislada como variante atípica del síndrome de Guillain-Barré tras sospecha
} de infección por SARS-CoV-2

\author{
Isolated facial diplegia as an atypical variant of Guillain-Barre syndrome after suspected SARS- \\ CoV-2 infection
}

\section{Diplegia facial isolada como uma variante atípica da síndrome de Guillain-Barré após suspeita de infecção por SARS-CoV-2}

\author{
Cristina Lavilla Olleros ${ }^{1}$, Marina López-Rubio², Chiara Fanciulli³, Adriana González-Munera4 ${ }^{4}$ Jesús Millán Núñez-Cortés³.
}

El síndrome de Guillain-Barré consiste en una afectación nerviosa que a menudo ocurre tras un proceso infeccioso y que normalmente se manifiesta como debilidad muscular o parálisis de inicio en ambas piernas con progresión ascendente, alteraciones de la sensibilidad y ausencia de reflejos osteotendinosos. Sin embargo, en ocasiones se presenta de forma atípica afectando sólo una región del organismo, como es el caso de la diplejía facial en la que se afectan ambos nervios faciales, que inervan la musculatura de la cara. En este caso se plantea que la infección por SARS CoV-2 pueda actuar como desencadenante de dicho síndrome, y concretamente en su forma atípica de diplejía facial.

\section{Conceptos clave:} ¿Qué se sabe sobre el tema?

La infección por SARS-CoV-2, responsable de la pandemia COVID-19, tiene repercusión multiorgánica, si bien la afectación respiratoria y gastrointestinal están más documentadas en la literatura. A nivel neurológico se han descrito múltiples expresiones clínicas, siendo el síndrome de Guillain-Barré una de ellas.

\section{¿Qué aporta este trabajo?}

Este caso se suma a los anteriores y refuerza la hipótesis de la relación entre la COVID-19 y el síndrome de Guillain-Barré. Además, se describe una variante regional, la diplejía facial, poco frecuente y poco conocida, pero que el clínico debe tener presente para poder orientar su diagnóstico y manejo.

1- Hospital General Universitario Gregorio Marañón. Servicio de Medicina Interna. Madrid, España. ORCID: https://orcid.org/00000002-3537-8607. E-mail de contacto: cristinalavillaolleros@gmail.com

2- Hospital General Universitario Gregorio Marañón. Servicio de Medicina Interna. Madrid, España. ORCID: https://orcid.org/0000 0001-8627-304X. E-mail de contacto: marinalopezrubio@outlook.com.

3- Hospital General Universitario Gregorio Marañón. Servicio de Medicina Interna. Madrid, España

4- Hospital General Universitario Gregorio Marañón. Servicio de Medicina Interna. Madrid, España. ORCID: https://orcid.org/00000002-4499-2799

Recibido: 2021-03-08 Aceptado: 2021-08-23

DOI: http://dx.doi.org/10.31053/1853.0605.v78.n4.32392

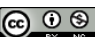

https://creativecommons.org/licenses/by-nc/4.0/

๑) Universidad Nacional de Córdoba

\section{Resumen:}

Introducción: Desde que se inició la pandemia por el SARS-CoV-2, se han descrito numerosos casos de síndrome de Guillain-Barré secundario a la COVID-19. Su presentación típica es la triada de parestesias, debilidad muscular ascendente y arreflexia, aunque hay diversas variantes regionales como la diplejía facial. Presentación del caso: Mujer de 35 años que, dos semanas después de un contacto estrecho con un caso confirmado de COVID-19, ingresa por miopericarditis probablemente viral, con estudio de autoinmunidad negativo, múltiples serologías virales negativas y positividad para IgG anti-SARS-CoV-2 con PCR negativa. Una semana tras el alta presenta paresia de ambos nervios faciales sin otras alteraciones neurológicas. Se realiza TAC craneal sin hallazgos y EMG que evidencia afectación bilateral de los nervios faciales. La paciente rechaza realización de punción lumbar. Discusión: La diplejía facial puede ocurrir en el contexto de diversas patologías, como tumores meníngeos o troncoencefálicos, agentes infecciosos, síndrome de Guillain-Barré, patologías autoinmunes, traumatismos, causas metabólicas o causas congénitas. En el caso descrito tras descartar mediante pruebas de imagen y analíticamente el resto de etiologías, y dada la presentación clínica, permanece como causa más probable el síndrome de GuillainBarré, posiblemente secundario a infección por SARS-CoV-2 dada la positividad de IgG antiSARS-CoV-2 tras un contacto con un caso confirmado. Conclusión: Este caso apoya la hipótesis de que la COVID-19 puede desencadenar el síndrome de Guillain-Barré, específicamente en forma de diplejía facial, una variante atípica que se debe conocer para su identificación y manejo precoz como parte de este síndrome.

Palabras clave: síndrome de Guillain-Barré; infecciones por coronavirus; parálisis facial; betacoronavirus.

\section{Abstract:}

Introduction: Since the SARS-CoV-2 pandemics began, multiple cases of Guillain-Barre syndrome secondary to COVID-19 have been described. Its typical presentation consists of the triad of paresthesia, ascending muscle weakness and areflexia, although there are several regional variants such as facial diplegia. Case presentation: Two weeks after a contact with a confirmed case of COVID-19, a 35-year-old woman presents with viral myopericarditis. Laboratory studies for autoimmune diseases come back negative, as well as multiple viral serologies. She presents anti-SARS-CoV-2 IgG, with negative PCR. A week after discharge she presents with palsy of both facial nerves, without other neurological abnormalities. She undergoes examination with cranial CT without findings, and an EMG which shows bilateral alteration of facial nerves. She refuses the performance of a lumbar puncture. Discussion: Facial diplegia can occur because of several illnesses, such as meningeal or brainstem tumors, infectious agents, Guillain-Barre syndrome, autoimmune diseases, trauma, metabolic causes or congenital causes. In our patient, having discarded other etiologies with imaging and analytical studies, the most probable cause is the Guillain-Barre syndrome. It is possibly secondary to SARS-CoV-2 infection given the presence of anti-SARS-CoV-2 IgG antibodies after contact with a confirmed case. Conclusion: This case supports the hypothesis that COVID-19 may trigger the Guillain-Barre syndrome, specifically as facial diplegia, which is an atypical variant that should be known to be early diagnosed and treated as part of this syndrome.

Keywords: Guillain-Barre syndrome; coronavirus infections; facial paralysis; betacoronavirus.

\section{Resumo:}

Introdução: Desde o início da pandemia SARS-CoV-2, numerosos casos de síndrome de Guillain-Barré secundária ao COVID-19 foram relatados. Sua apresentação típica é a tríade de parestesia, fraqueza muscular ascendente e arreflexia, embora existam muitas variantes regionais, como a diplegia facial. Relato do caso: Mulher de 35 anos que, duas semanas após contato com um caso confirmado de COVID-19, é internada devido a miopericardite de provável etiologia viral, com um estudo de autoimunidade negativo, múltiplas sorologias virais negativas e IgG positiva para SARS-CoV-2 com PCR negativo. Uma semana após a alta, apresentou paresia de ambos os nervos faciais sem outras alterações neurológicas. Uma tomografia computadorizada de crânio foi realizada sem achados e um EMG mostrando envolvimento bilateral dos nervos faciais. O paciente se recusa a realizar uma punção lombar. Discussão: A diplegia facial pode ocorrer no contexto de várias patologias, como tumores meníngeos ou do tronco encefálico, agentes infecciosos, síndrome de Guillain-Barré, patologias autoimunes, traumas, causas metabólicas ou congênitas. No caso descrito, após a exclusão das demais etiologias por exames laboratoriais e de imagem, e dada a apresentação clínica, a síndrome de Guillain-Barré continua sendo a causa mais provável, possivelmente secundária à infecção por SARS-CoV-2 pela positividade de IgG-SARS-CoV-2 após contato com um caso confirmado. Conclusão: O presente caso corrobora a hipótese de que a COVID19 pode desencadear a síndrome de Guillain-Barré, especificamente na forma de diplegia facial, uma variante atípica que deve ser reconhecida para o diagnóstico e tratamento precoces dessa síndrome.

Palavras-chave: síndrome de Guillain-Barré; infecções por coronavirus; paralisia facial; betacoronavirus. 


\section{INTRODUCCIÓN}

La diplejía facial consiste en la parálisis simultánea de ambos nervios faciales. Es una entidad poco frecuente y representa menos del $2 \%$ de casos de parálisis facial. Se puede encontrar asociada a patologías como el síndrome de Guillain-Barré, tumores o infecciones, entre otras ${ }^{(1)}$.

El síndrome de Guillain-Barré es una polirradiculopatía inmunomediada caracterizada por la desmielinización segmentaria y presencia de infiltrados inflamatorios en el endoneuro y vainas de mielina ${ }^{(2)}$. La etiología es desconocida, pudiendo jugar un papel importante las infecciones virales. La mitad de los pacientes con síndrome de Guillain-Barré presenta previamente un cuadro infeccioso, normalmente gastrointestinal o de vías respiratorias altas. La presentación clínica típica es la tríada de parestesias, debilidad muscular ascendente y arreflexia ${ }^{(2)}$. Sin embargo, se han descrito múltiples variantes atípicas como la diplejía facial, siendo importante identificarlas como tal ya que en ocasiones son la forma de presentación de formas graves que se beneficiarían de tratamiento específico ${ }^{(1-4)}$.

La enfermedad por coronavirus de 2019 (COVID-19) está producida por el coronavirus de tipo 2 causante del síndrome respiratorio agudo severo (SARS-CoV-2), y sus primeros casos se describen en Wuhan, China, en diciembre de 2019. Dada su rápida expansión global, la Organización Mundial de la Salud declara la COVID-19 como pandemia el 11 de marzo de $2020^{(3)}$. Poco más de un año después, a 18 de marzo de 2021, se han declarado en el mundo más de 120.000.000 casos, con en torno a 2,6 millones de fallecimientos ${ }^{(5)}$. Su presentación más habitual es con síntomas respiratorios y gastrointestinales. Sin embargo, se han descrito también manifestaciones neurológicas, entre ellas el síndrome de Guillain-Barré(3)

\section{PRESENTACIÓN DEL CASO}

Mujer de 35 años con antecedentes de hipertensión arterial y dos episodios de preeclampsia. Acude a su centro de salud por clínica sugestiva de COVID-19 tras contacto con un caso positivo, con seguimiento ambulatorio. Dos semanas después acude a urgencias por fiebre de hasta $39,5^{\circ} \mathrm{C}$, despeños diarreicos, cefalea holocraneal y dolor centrotorácico de características pleuríticas de 5 días de evolución. No refiere vacunaciones recientes. A la exploración física destaca regular estado general y taquicardia. Analíticamente destaca anemia microcítica hipocrómica y elevación de reactantes de fase aguda (fibrinógeno $>1000 \mathrm{mg} / \mathrm{dL}$, proteína C reactiva $36.2 \mathrm{mg} / \mathrm{dL}$, velocidad de sedimentación globular $1^{\text {a }}$ Hora $87 \mathrm{~mm}$ ), Nt-proBNP $3200 \mathrm{ng} / \mathrm{L}$, troponina I $30 \mathrm{ng} / \mathrm{L}$ y dímero D 750 $\mathrm{ng} / \mathrm{ml}$. Se realiza angio-tomografía axial computarizada (TAC) que sugiere engrosamiento pericárdico. Esto, junto a la clínica y elevación de enzimas de daño miocárdico, apoya el diagnóstico de miopericarditis. Se realiza ecocardiograma descartando disfunción ventricular o derrame pericárdico. Estudio de autoinmunidad negativo y múltiples serologías virales negativas para afectación aguda. PCR para SARS-CoV-2 negativa, si bien con positividad para anticuerpos IgG anti-SARS-CoV-2. Se inicia tratamiento con piperacilina-tazobactam, colchicina y antiinflamatorios no esteroideos ante el diagnóstico de miopericarditis probablemente de origen viral, consiguiendo mejoría clínica y analítica y pudiendo dar de alta a la paciente.

Una semana después acude a revisión, refiriendo dificultad para determinados movimientos faciales (sonreír, dar besos, emitir sonidos labiales), lagrimeo bilateral y acorchamiento en la punta de la lengua. Audición y gusto conservados, movilidad y sensibilidad de extremidades normal. Niega problemas deglutorios o diplopía. A la exploración se observa paresia del VII par craneal bilateral (disminución de fuerza al fruncir la frente, cerrar los párpados, elevar las cejas e imposibilidad para sonreír y fruncir los labios). Resto de pares craneales sin alteraciones. Fuerza conservada en miembros superiores e inferiores, con reflejos presentes y vivos a todos los niveles. Sensibilidad exteroceptiva y propioceptiva conservadas. Marcha normal. Romberg negativo. Analíticamente se observa trombocitosis aislada. Se realiza TAC craneal sin alteraciones. Se propone realización de punción lumbar, pero la paciente la rechaza y solicita alta voluntaria sin poder completar el estudio. Acude a consulta la semana siguiente con resolución parcial de la clínica, persistiendo paresia facial periférica de predominio izquierdo sin otros datos de focalidad neurológica. Analíticamente trombocitosis en descenso. Dos semanas después se realiza electromiograma (EMG) para estudio del reflejo del parpadeo tras estimulación del nervio supraorbitario. Bilateralmente se observan una respuesta temprana (R1) ipsilateral y respuestas intermedias (R2) ipsilateral y contralateral de latencia prolongada. Es decir, se objetivan leves cambios neurogénicos en la musculatura del nervio facial bilateral, compatibles con afectación bilateral de la eferencia (nervio facial bilateral). Tras la instauración de la paresia se continua con colchicina como único tratamiento. En la última visita, aproximadamente tres semanas tras el inicio del cuadro, la clínica había remitido casi por completo.

\section{DISCUSIÓN}

La afectación simultánea en forma de parálisis de ambos nervios faciales, en un tiempo inferior a 4 semanas, se denomina diplejía facial y representa menos del $2 \%$ de casos de parálisis facial (1). Puede producirse como respuesta a múltiples causas, entre las que destacan: síndrome de Guillain-Barré, tumores meníngeos y troncoencefálicos, agentes infecciosos (Borrelia, sífilis, VIH, VHS, VEB o tuberculosis) y una forma idiopática (parálisis de Bell). Además, se han descrito casos asociados a etiologías menos frecuentes como sarcoidosis, traumatismos craneoencefálicos, linfoma, leucemia, causas metabólicas como la diabetes o neuropatía alcohólica y causas congénitas como el síndrome de Moebius ${ }^{(1,4,6-8)}$

En el caso descrito, la realización de una TAC craneal sin alteraciones descarta la etiología tumoral o hemorrágica. La ausencia de adenopatías en la radiografía de tórax junto con niveles normales de la enzima convertidora de angiotensina reduce la probabilidad de sarcoidosis. Otras patologías se descartan ante resultados negativos del exhaustivo análisis de despistaje autoinmune.

En cuanto a la etiología infecciosa, la paciente presenta negatividad para IgM de Borrelia, serología luética, rosa de Bengala y cuantiferon, descartando respectivamente enfermedad de Lyme, sífilis, brucelosis y tuberculosis. Asimismo, se realizan serologías de múltiples virus con resultado negativo.

Una vez descartadas otras etiologías y por la clínica de la paciente, queda como diagnóstico más probable el síndrome de GuillainBarré. La afectación facial es habitual, aunque la presentación en forma de diplejía facial aislada ocurre en menos del $1 \%$ de los $\operatorname{casos}^{(4)}$. Este caso cumple algunos de los criterios diagnósticos para esta variante regional: diplejía facial en ausencia de oftalmoplejía, ataxia y debilidad de extremidades; curso monofásico con intervalo entre el inicio de los síntomas y el nadir de entre 12 horas y 28 días seguido de una fase de meseta; antecedente de sintomatología infecciosa las 3 semanas previas; y evidencia electrofisiológica de neuropatía ${ }^{(9)}$. Puesto que la paciente rechaza la realización de punción lumbar para estudio del líquido cefalorraquídeo (LCR) no se puede comprobar la disociación albúminocitológica.

A pesar de no haber obtenido PCR positiva para SARS-CoV-2, la presentación de sintomatología sugerente de dicha infección dos semanas antes, junto con el contacto con un caso positivo referido por la paciente y la presencia al ingreso de serología positiva para SARS-CoV-2, apoyan en esta paciente el diagnóstico de infección por SARS-CoV-2 reciente, que se plantea como posible desencadenante de los cuadros posteriores. Se han descrito algunos casos de afectación cardíaca por este virus, sobre todo elevación de enzimas de daño miocárdico y arritmias, aunque también hay casos descritos de miopericarditis aislada, como sería el caso de nuestra paciente ${ }^{(10-11)}$. Asimismo, se han descrito 
numerosas manifestaciones neurológicas secundarias al COVID-19, con afectación tanto del sistema nervioso central como del sistema nervioso periférico y el músculo esquelético ${ }^{(12)}$ La mayoría de casos de diplejía facial se describe en el contexto de pacientes con diagnóstico de síndrome de Guillain-Barré secundario a la infección por SARS-CoV-2 ${ }^{(13)}$. En la mayoría de casos la diplejía facial se presenta junto con otra clínica neurológica, pero ya se ha descrito un posible caso de síndrome de Guillain-Barré en forma de variante regional con diplejía facial aislada en un paciente infectado por SARS-CoV-2 ${ }^{(14)}$ También hay casos descritos de diplejía facial y otras neuropatías de pares craneales probablemente secundarias al SARS-CoV-2 en ausencia de síndrome de Guillain Barré, aunque el número de casos publicados es menor ${ }^{(15-16)}$.

En base a lo expuesto, tras descartar otras etiologías y ante clínica y pruebas complementarias compatibles a pesar de no haber podido demostrar la disociación albuminocitológica en el LCR, la paciente descrita se diagnosticó de diplejía facial en el contexto de probable síndrome de Guillain-Barré secundario a infección por SARS-CoV-2.

\section{CONCLUSIONES}

La diplejía facial es un síndrome poco frecuente y cuyo origen puede ser muy variado, siendo uno de los más frecuentes el síndrome de Guillain-Barré. Este caso se suma a los descritos en la literatura en la afirmación de que la infección por SARS-CoV-2 puede desencadenar dicho síndrome, aunque harán falta más casos y estudios para poder afirmarlo con certeza. En cualquier caso, el clínico debe conocer esta posible complicación para identificarla si aparece y ser precoz en su manejo.

\section{Limitaciones de responsabilidad:}

La responsabilidad de este trabajo es exclusivamente de los autores.

Conflicto de interés:

Ninguno

\section{Fuentes de apoyo:}

La presente investigación no contó con fuentes de financiación.

\section{Originalidad:}

Este artículo es original y no ha sido enviado para su publicación a otro medio de difusión científica en forma completa ni parcialmente.

\section{Cesión de derechos:}

Los participantes de este trabajo ceden el derecho de autor a la Universidad Nacional de Córdoba para publicar en la RFCM y realizar las traducciones necesarias.

\section{Contribucion de los autores:}

Todos los autores han participado en la concepción del diseño, recolección de la información y elaboración del manuscrito, haciéndose públicamente responsables de su contenido y aprobando su versión final.

\section{BIBLIOGRAFÍA}

1. Marzo Sola M, Vaquero Garrido M, Bártulos Iglesias M, Gil Pujades A. Variante facial del síndrome de Guillain-Barré en un paciente, días después de vacunarse de la gripe A. Neurología. 2011 Apr;26(3):186-8. doi: 10.1016/j.nrl.2010.05.009.

2. Casademont Pou JM, Rojas García R. Enfermedades de los nervios periféricos. In: Farreras Rozman Medicina Interna, vol. II. Barcelona: Elsevier; 2016. p. 1470

3. Agosti E, Giorgianni A, D'Amore F, Vinacci G, Balbi S, Locatelli $D$. Is Guillain-Barrè syndrome triggered by SARS-CoV-2? Case report and literature review. Neurol Sci. 2021 Feb;42(2):607-612. doi: 10.1007/s10072-020-04553-9.

4. Lowe J, Pfaff J. The Ultimate Poker Face: A Case Report of Facial Diplegia, a Guillain-Barré Variant. Clin Pract Cases Emerg Med. 2020 Apr 23:4(2):150-153. doi: 10.5811/cpcem.2020.2.45556.
5. España. Ministerio de Sanidad. Centro de Coordinación de Alertas y Emergencias Sanitarias. Actualización no 335. Enfermedad por el coronavirus (COVID-19). 18.03.2021. Disponible en: https://www.mscbs.gob.es/profesionales/saludPublica/ccayes/alerta sActual/nCov/documentos/Actualizacion 335 COVID-19.pdf

6. Keane JR. Bilateral seventh nerve palsy: analysis of 43 cases and review of the literature. Neurology. 1994 Jul;44(7):1198-202. doi: 10.1212/wnl.44.7.1198.

7. Varol S, Ozdemir HH, Akil E, Arslan D, Aluclu MU, Demir CF, Yucel Y. Facial diplegia: etiology, clinical manifestations, and diagnostic evaluation. Arq Neuropsiquiatr. 2015 Dec;73(12):9981001. doi: 10.1590/0004-282X20150174.

8. Pothiawala S, Lateef F. Bilateral facial nerve palsy: a diagnostic dilemma. Case Rep Emerg Med. 2012;2012:458371. doi: 10.1155/2012/458371.

9. Wakerley BR, Yuki N. Isolated facial diplegia in Guillain-Barré syndrome: Bifacial weakness with paresthesias. Muscle Nerve. 2015 Dec;52(6):927-32. doi: 10.1002/mus.24887.

10. Kumar R, Kumar J, Daly C, Edroos SA. Acute pericarditis as a primary presentation of COVID-19. BMJ Case Rep. 2020 Aug 18;13(8):e237617. doi: 10.1136/bcr-2020-237617.

11. Tung-Chen Y. Acute pericarditis due to COVID-19 infection: An underdiagnosed disease? Med Clin (Barc). 2020 Jul 10;155(1):4445. doi: 10.1016/j.medcli.2020.04.007.

12. Mao L, Jin H, Wang M, Hu Y, Chen S, He Q, Chang J, Hong $C$, Zhou Y, Wang D, Miao X, Li Y, Hu B. Neurologic Manifestations of Hospitalized Patients With Coronavirus Disease 2019 in Wuhan, China. JAMA Neurol. 2020 Jun 1;77(6):683-690. doi: 10.1001/jamaneurol.2020.1127.

13. Abolmaali M, Heidari M, Zeinali M, Moghaddam P, Ramezani Ghamsari M, Jamshidi Makiani M, Mirzaasgari Z. Guillain-Barré syndrome as a parainfectious manifestation of SARS-CoV-2 infection: A case series. J Clin Neurosci. 2021 Jan;83:119-122. doi: 10.1016/j.jocn.2020.11.013.

14. Juliao Caamaño DS, Alonso Beato R. Facial diplegia, a possible atypical variant of Guillain-Barré Syndrome as a rare neurological complication of SARS-CoV-2. J Clin Neurosci. 2020 Jul;77:230-232. doi: 10.1016/j.jocn.2020.05.016.

15. Costello F, Dalakas MC. Cranial neuropathies and COVID-19: Neurotropism and autoimmunity. Neurology. 2020 Aug 4;95(5):195196. doi: 10.1212/WNL.0000000000009921.

16. Paliwal VK, Garg RK, Gupta A, Tejan N. Neuromuscular presentations in patients with COVID-19. Neurol Sci. 2020 Nov;41(11):3039-3056. doi: 10.1007/s10072-020-04708-8. 